Vorstandswahlen der

\title{
Arbeitsgemeinschaften der DRG auf dem 95. Deutschen Röntgenkongress, 28. - 31. Mai 2014 in Hamburg
}

Auf dem diesjährigen Röntgenkongress finden für folgende Arbeitsgemeinschaften Vorstandswahlen statt:

- AG Gastrointenstinal-/Abdominaldiagnostik

- AG Herzdiagnostik

- AG Informationstechnologie @GIT

- AG Methodik und Forschung (AGMF)

- AG Mammadiagnostik

- AG Physik und Technik

- AG Thoraxdiagnostik

Entsprechend der Arbeitsordnung der Arbeitsgemeinschaften bedürfen die zur Wahl vorgeschlagenen Kandidaten der Zustimmung des Vorstandes der DRG (nihil obstat). Die Mitglieder der oben genannten Arbeitsgemeinschaften werden hiermit eingeladen, Kandidaten für den Vorstand der Wahlperiode 2014-2016 vorzuschlagen. Die Vorschläge müssen schriftlich eingereicht werden und sollten das Einverständnis der vorgeschlagenen Kandidaten enthalten.

\section{AG Gastrointenstinal-|Abdominal- diagnostik \\ $\checkmark$}

Der Vorstand der AG Gastrointenstinal-/ Abdominaldiagnostik bittet seine Mitglieder um Kandidatenvorschläge für die Vorstandswahl 2014. Als Kandidaten können nur Mitglieder vorgeschlagen werden, die am 1. März 2014 ordentliche Mitglieder der Arbeitsgemeinschaft waren. Vorschläge bedürfen der Schriftform und sind bis spätestens 12. März 2014 an den Vorstand der Arbeitsgemeinschaft zu Händen des Vorsitzenden, Prof. Dr. med. Andreas Schreyer, Regensburg, oder an die Geschäftsstelle der DRG z. Hd. Frau Müller zu senden.

\section{AG Herzdiagnostik \\ $\nabla$}

Der Vorstand der AG Herzdiagnostik bittet seine Mitglieder um Kandidatenvorschläge für die Vorstandswahl 2014. Als Kandidaten können nur Mitglieder vorgeschlagen werden, die am 1. März 2014 ordentliche Mitglieder der Arbeitsgemeinschaft waren. Vorschläge bedürfen der Schriftform und sind bis spätestens 12. März 2014 an den Vorstand der Arbeitsgemeinschaft zu Händen des Vorsitzenden, Prof. Barkhausen, Tübingen, oder an die Geschäftsstelle der DRG z. Hd. Herrn Völker zu senden.

\section{AG Informationstechnologie @GIT $\nabla$}

Der Vorstand der AG Informationstechnologie@GIT bittet seine Mitglieder um Kandidatenvorschläge für die Vorstandswahl 2014. Als Kandidaten können nur Mitglieder vorgeschlagen werden, die am 1. März 2014 ordentliche Mitglieder der Arbeitsgemeinschaft waren. Vorschläge bedürfen der Schriftform und sind bis spätestens 12. März 2014 an den Vorstand der Arbeitsgemeinschaft zu Händen des Vorsitzenden Prof. Dr. med. Elmar Kotter, Freiburg, oder an die Geschäftsstelle der DRG z. Hd. Herrn Völker zu senden.

\section{AG Methodik und Forschung (AGMF) $\nabla$}

Der Vorstand der AG Methodik und Forschung (AGMF) bittet seine Mitglieder um Kandidatenvorschläge für die Vorstandswahl 2014. Als Kandidaten können nur Mitglieder vorgeschlagen werden, die am 1. März 2014 ordentliche Mitglieder der Arbeitsgemeinschaft waren. Vorschläge bedürfen der Schriftform und sind bis spätestens 12. März 2014 an den Vorstand der Arbeitsgemeinschaft zu Händen des Vorsitzenden Dr. Michel Eisenblätter, Münster, oder an die Geschäftsstelle der DRG z. Hd. Herrn Völker zu senden.

\section{AG Mammadiagnostik}

\section{$\nabla$}

Der Vorstand der AG Mammadiagnostik bittet seine Mitglieder um Kandidatenvorschläge für die Vorstandswahl 2014. Als Kandidaten können nur Mitglieder vorgeschlagen werden, die am 1 . März 2014 ordentliche Mitglieder der Arbeitsgemeinschaft waren. Vorschläge bedürfen der Schriftform und sind bis spätestens 12. März 2014 an den Vorstand der Arbeitsgemeinschaft zu Händen des Vorsitzenden Prof. Dr. med. Ulrich Bick, Berlin oder an die Geschäftsstelle der DRG z. Hd. Frau Engelhardt zu senden.

\section{AG Physik und Technik \\ $\nabla$}

Der Vorstand der AG Physik und Technik bittet seine Mitglieder um Kandidatenvorschläge für die Vorstandswahl 2014. Als Kandidaten können nur Mitglieder vorgeschlagen werden, die am 1 . März 2014 ordentliche Mitglieder der Arbeitsgemeinschaft waren. Vorschläge bedürfen der Schriftform und sind bis spätestens 12. März 2014 an den Vorstand der Arbeitsgemeinschaft zu Händen des Vorsitzenden Dipl.-Phys. Horst Lenzen, Münster oder an die Geschäftsstelle der DRG z. Hd. Herrn Völker zu senden.

\section{AG Thoraxdiagnostik \\ $\nabla$}

Der Vorstand der AG Thoraxdiagnostik bittet seine Mitglieder um Kandidatenvorschläge für die Vorstandswahl 2014. Als Kandidaten können nur Mitglieder vorgeschlagen werden, die am 1 . März 2014 ordentliche Mitglieder der Arbeitsgemeinschaft waren. Vorschläge bedürfen der Schriftform und sind bis spätestens 12. März 2014 an den Vorstand der Arbeitsgemeinschaft zu Händen des Vorsitzenden Prof. Dr. C. P. Heußel, Heidelberg, oder an die Geschäftsstelle der DRG z. Hd. Frau Engelhardt zu senden.

\section{Vorstandswahlen der DeGIR}

(Deutsche Gesellschaft für Interventionelle Radiologie) auf dem 95. Deutschen Röntgenkongress 2014, 28. - 31. Mai 2014 in Hamburg $\nabla$

Der Vorstand der DeGIR (Deutsche Gesellschaft für Interventionelle Radiologie) bittet seine Mitglieder um Kandidatenvorschläge für die Vorstandswahl 2014. Als Kandidaten können nur Mitglieder vorgeschlagen werden, die am 1 . März 2014 ordentliche Mitglieder der DeGIR waren. Vorschläge bedürfen der Schriftform und sind bis spätestens 12. März 2014 an den Vorstand der DeGIR zu Händen des Vorsitzenden Prof. Dr. med. Dierk Vorwerk, Ingolstadt, oder an die Geschäftsstelle der DeGIR z. Hd. Frau Epsch zu senden. 